\title{
Efectos de los anuncios de política monetaria y la credibilidad sobre las expectativas de inflación: evidencia para Colombia
}

\author{
Effects of Monetary Policy Announcements and Credibility \\ on Inflation Expectations: Evidence for Colombia
}

Efeitos dos anúncios de política monetária e credibilidade sobre as expectativas de inflação: evidência para a Colômbia

DOI: https://doi.org/10.19053/01203053.v38.n67.2019.7912

Fecha de recepción: 6 de abril de 2018

Fecha de aceptación: 31 de octubre de 2018

Cómo citar este artículo/ To reference this article / Comment citer cet article / Para citar este artigo:

Galvis, J. C. \& Anzoategui-Zapata, J. (2019). Efectos de los anuncios de política monetaria y la credibilidad sobre las expectativas de inflación: evidencia para Colombia. Apuntes del Cenes, 38(67). https://doi. org/10.19053/01203053.v38.n67.2019.7912

\footnotetext{
* PhD. en Economía. Profesor Universidad Pontificia Bolivariana, Medellín, Colombia. jcgalvisciro@gmail.com (1) http://orcid. org/0000-0001-6680-275X

** Msc en Economía. Profesor Universidad Autónoma Latinoamericana, Medellín, Colombia. juan.anzoategui@unaula.edu.co (D) https://orcid.org/0000-0003-0588-1364
} 


\section{Resumen}

Este documento analiza los efectos de los anuncios del banco central sobre las expectativas de inflación para la economía colombiana. En particular, son estudiados los impactos de los pronunciamientos sobre la tasa de interés y los choques macroeconómicos sobre las tasas de inflación compensadas, dado el escenario de credibilidad de la política monetaria. La evidencia empírica es abordada por medio de un análisis de regresión con un modelo EGARCH para el período 2008-2016. Los resultados muestran que las expectativas de inflación responden a los anuncios de política. Además, los pronunciamientos fueron más efectivos en anclar las expectativas durante el período 2012-2016 y la mayor credibilidad de la política permitió que las expectativas fueran más estables frente a los choques externos.

Palabras clave: banco central, expectativas de inflación, política monetaria, modelización econométrica.

Clasificación JEL: C5, C51, E52, E58, E61. 


\section{Abstract}

This paper refers to the literature on the effects of central bank announcements on inflation expectations in developing countries. In particular, based on the Colombian experience, we study the impact of interest rate announcements and macroeconomic shocks on breakeven inflation rates, given the monetary policy credibility scenario. The empirical evidence is implemented by regression analysis using an EGARCH model between 2008-2016. We find that inflation expectations respond to policy statements in Colombia. Our results show that the central bank pronouncements were more effective in anchoring expectations in the period 2012-2016 and the greater monetary policy credibility allows inflation expectations to be more stable before external shocks.

Keywords: central bank, inflation expectations, monetary policy, econometric modeling. 


\section{Resumo}

Este trabalho analisa os efeitos dos anúncios do banco central sobre as expectativas de inflação para a economia colombiana. Em particular, são avaliados os efeitos dos anúncios da taxa de juros e choques macroeconômicos sobre as taxas de inflação compensadas em diferentes cenários de credibilidade da política monetária. A evidência empírica é abordada por meio de analises de regressão com um modelo EGARCH para o período 2008-2016. Os resultados mostram que as expectativas de inflação reagem aos anúncios de política. Além disso, os anúncios do banco central foram mais eficaz para ancorar as expectativas durante o período 2012-2016 e sua maior credibilidade na política monetária permitiu que as expectativas foram mais estáveis frente a choques externos.

Palavras-chave: banco central, expectativas de inflação, política monetária, modelagem econométrica. 


\section{INTRODUCCIÓN}

Las decisiones de política monetaria dependen, de forma crítica, de qué tanto estén las expectativas de inflación ancladas en las metas de inflación anunciadas por el banco central (Bernanke, Laubach, Mishkin \& Posen, 1999). La correcta administración de las expectativas incrementa la eficiencia de la política monetaria y suministra una señal de desempeño del régimen de metas de inflación como un esquema monetario (Woodford, 2005). En la práctica, la comunicación es una herramienta de política que tiene el banco central para coordinar las expectativas y mover los mercados financieros (Blinder, Ehrmann, Fratzcher, De Haan \& Jansen, 2008). En particular, las decisiones sobre la tasa de intervención y la credibilidad en los objetivos del banco central tienen el potencial para afectar la velocidad de convergencia de las expectativas hacia las metas de inflación (Gürkaynak, Levin \& Swanson, 2010).

En el régimen de metas de inflación, los bancos centrales se comunican de forma periódica con el público a través de varios canales y diferentes estrategias (Ehrmann \& Fratzscher, 2007). La comunicación ofrece información clave sobre el entorno económico y suministra importantes pistas sobre las razones que soportan las decisiones de política monetaria (Born, Ehrmann \& Fratzscher 2014). Desde el año 2000, la literatura sobre la comunicación de los bancos centrales y la administración de las expectativas ha crecido. En general, la evidencia empírica sugiere 
que el régimen de metas de inflación es un poderoso esquema para anclar las expectativas como consecuencia de las metas de inflación, la independencia del banco, la transparencia y la credibilidad asociada (Johnson, 2003; Levin, Natalucci \& Piger, 2004; Gürkaynak et al., 2010).

Para economías desarrolladas existen varios estudios. En el caso de Europa, Jansen y De Haan (2007) encuentran que la comunicación del Banco Central Europeo relacionada con la vigilancia a la estabilidad de precios ayuda a estabilizar las expectativas de inflación. Otro resultado importante, reportado por Ehrmann, Fratzscher, Gürkaynak y Swanson (2011), sugiere que la unificación del mercado de deuda pública en la Unión Europea ha permitido la convergencia y el anclaje de las expectativas en Europa. Entre el período 2001-2007 las expectativas de inflación permanecieron sin grandes cambios en Estados Unidos y Europa (Clark \& Nakata, 2008). Sin embargo, diversos choques han comenzado a afectar las expectativas. Existen estudios que han evaluado el efecto de la crisis financiera de 2007-2008 en las expectativas de inflación. Entre estos, Galati, Poelhekke y Zhou (2011) muestran que la crisis tuvo efectos significativos sobre el anclaje de las expectativas de inflación en Estados Unidos, la Unión Europea y el Reino Unido. Además, las reacciones de las expectativas de inflación a las noticias de política monetaria y las noticias macroeconómicas han aumentado desde 2006. Por otra parte, Moessner (2015) analiza la política de relajamiento monetario en Estados Unidos luego de la crisis financiera de 2007. Sus resultados muestran que la política de forward guidance de la Reserva Federal ha tenido pocos efectos en el anclaje de las expectativas de inflación en Estados Unidos.

Los estudios respecto a los efectos de las expectativas de inflación son escasos para economías en desarrollo. Entre los estudios que existen, De Pooter, Robitaille, Walker y Zdinak (2014) sugieren que las expectativas de inflación en Brasil, Chile y México muestran una importante respuesta a las noticias macroeconómicas y las expectativas no están ancladas con firmeza, a pesar de que estas economías cuentan con el régimen de metas de inflación adoptado varios años atrás. Sumado a esto, Montes, Oliveira, Curi y Nicolay (2016) señalan que la mayor claridad y transparencia en la comunicación del Banco Central de Brasil reduce los desacuerdos en las expectativas de inflación.

Para economías emergentes que han adoptado el régimen de metas de inflación, existen dos hechos relevantes que deben ser considerados. Primero, la política monetaria es administrada 
en escenarios donde no hay una credibilidad consolidada en los objetivos del banco central. Segundo, el anclaje adecuado de las expectativas de inflación es demorado porque los mercados están aún en un proceso de aprendizaje sobre los objetivos de la política monetaria. Como resultado, diversas variables pueden desestabilizar las expectativas de inflación en economías emergentes. El principal objetivo de este estudio es analizar los efectos de la comunicación sobre las expectativas de inflación, bajo diferentes escenarios de credibilidad. En particular, presentamos evidencia empírica para orientar este problema hacia la economía colombiana. El análisis para Colombia es relevante, porque es una economía emergente que trabaja con el régimen de metas de inflación y ha logrado importantes metas macroeconómicas (regla fiscal, grado de inversión, inflaciones menores a un dígito). Los resultados de este estudio permiten a los formuladores de política diseñar mejores planes para la política monetaria e indica las consecuencias que trae el desempeño de la credibilidad sobre las estrategias de comunicación del banco central.

El resto del documento está organizado así: en la sección 2 se desarrolla la metodología para analizar los efectos de los anuncios sobre las expectativas. En la sección 3 se suministra evidencia empírica, por medio de un análisis econométrico, sobre los efectos de los anuncios y los choques macroeconómicos en las expectativas de inflación. En la sección 4 se dividen los resultados con base en los escenarios de credibilidad de la política monetaria. Por último, en la sección 5 se exponen las conclusiones.

\section{METODOLOGÍA Y DATOS}

Las expectativas orientan las decisiones de inversión y son, por tanto, críticas para el desempeño macroeconómico. Entre las diferentes expectativas que tienen los agentes, el comportamiento de las expectativas de inflación es importante porque indica el nivel de confianza del público en el banco central y suministra información relevante sobre la credibilidad de la política monetaria. En consecuencia, el seguimiento de las expectativas es fundamental para que los formuladores de política adopten una postura (Mankiw, Reis \& Wolfers, 2003; Levin et al., 2004; De Mendonça, 2007; Montes et al., 2016).

Como medida de las expectativas de inflación son utilizadas las tasas de inflación compensadas o Break-Even Inflation rates (BEI). Las tasas de inflación compensadas son una medida de mercado de las expectativas de inflación y valoran el cubrimiento por inflación esperada que los inversionistas demandan en el mercado de deuda 
pública (Gürkaynak et al., 2010). Estas tasas de inflación compensadas son calculadas como la tasa de inflación que hace a un inversionista indiferente entre un bono nominal y un bono indexado a inflación. Es decir,

$$
\pi_{t}^{e}=f_{(t, \text { nominal })}^{m}-f_{(t, \text { index })}^{m}
$$

Donde $\pi_{t}^{e}$ son las expectativas de inflación, $f_{(t, n o m i n a l)}{ }^{m}$ es el retorno del bono nominal, $f_{(t, \text { index })}^{m}$ es el retorno del bono indexado y $\mathrm{m}$ es la madurez en años del bono. De esta forma, la diferencia en el retorno entre ambos bonos es atribuida a la inflación esperada por los inversionistas. Una importante ventaja de este tipo de medida de las expectativas de inflación es que tiene una frecuencia mayor que otros indicadores y permite contar con una medida más certera para estimar los impactos de los anuncios de política monetaria (ver, por ejemplo, Kliesen \& Schmid, 2004, Jansen \& De Haan, 2007, Gürkaynak et al., 2010, Galati et al., 2011, Moessner, 2015). En el caso de Colombia, las tasas de inflación compensadas han probado ser una medida eficiente de las expectativas de inflación (Vargas, González, González, Romero \& Rojas, 2010). Por tanto, en este estudio son utilizados títulos con madurez a 1,5 y 10 años con el fin de calcular las expectativas de inflación para 1, 5 y 10 años (ver Figura 1).

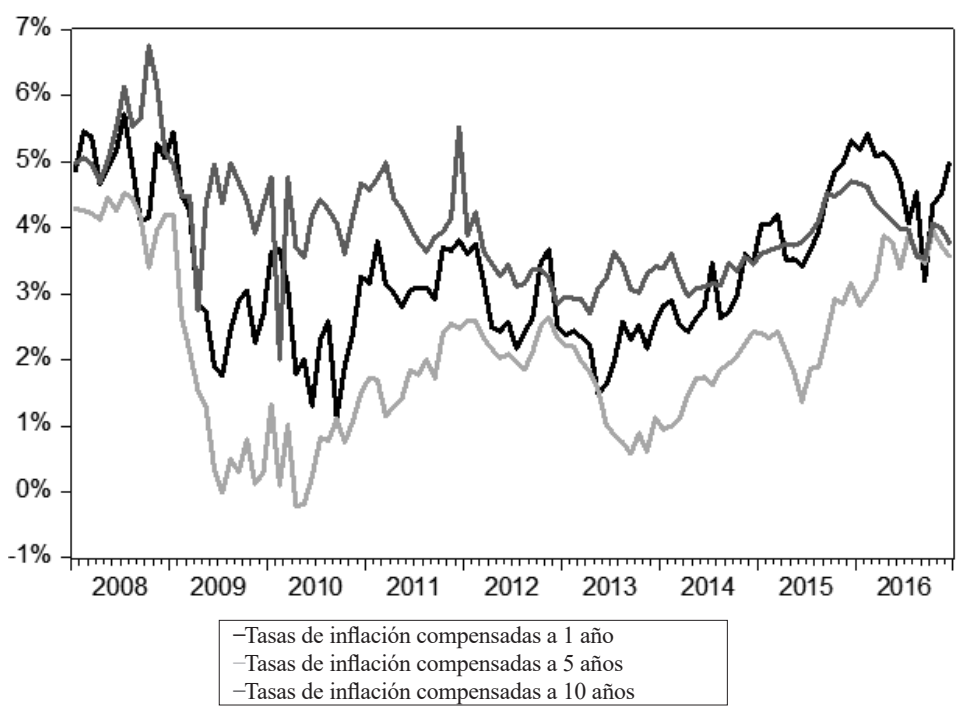

Figura 1. Tasas de inflación compensadas a 1, 5 y 10 años.

Fuente: elaboración propia con base en datos del banco central de Colombia (Banco de la República). 
Los anuncios de la tasa de interés del banco central revelan información importante sobre los riesgos que afectan la estabilidad de precios y el desempeño económico. Como resultado, los anuncios del banco central tienen la capacidad para guiar las expectativas de inflación (Eijffinger \& Geraats, 2006; Blinder et al., 2008; Jansen, 2011). Los mercados financieros fijan el precio de los activos en una conducta de planeación hacia adelante (forward-looking) y no responden a los componentes esperados de los anuncios del banco central. Es decir, debido a que solo el componente inesperado de los anuncios del banco contiene nueva información es aquel el que afecta las expectativas de los mercados (Jansen \& De Haan, 2007; Ehrmann \& Fratzscher, 2007). El componente sorpresa o inesperado (MPS) de los anuncios de la tasa de política es definido de forma usual en los estudios empíricos, como los cambios en las tasas de interés a 30 días en los días en que el banco central realiza sus anuncios sobre la tasa de interés (Kuttner, 2001 y Blinder et al., 2008) ${ }^{1}$. Por tanto, el siguiente modelo básico es considerado para el análisis empírico:

$$
\Delta \pi_{t}^{e}=\alpha_{0}+\alpha_{1} M P S_{t}+\varepsilon_{t}^{0}
$$

$$
\Delta \pi_{t}^{e}=\alpha_{2}+\alpha_{3} M P S_{t}+\alpha_{4} X_{t}+\varepsilon_{t}^{l}[3]
$$

Donde $\Delta \pi_{t}^{e}$ son los cambios en las expectativas de inflación entre $t+1 \mathrm{y} t$, siendo $t$ el día del anuncio del banco central. MPS es el componente inesperado en los anuncios de la tasa de interés y $X$ es un vector de variables control calculadas durante los días de los anuncios del banco central.

Los residuales son $\varepsilon_{t}=v_{t} h_{t} \sim\left(0, h_{t}^{2}\right)$ con $v_{t}=\left(\varepsilon_{t} / h_{t}\right) \sim i i d(0,1)$. En concreto, se asume que los residuales tienen una varianza condicional con el siguiente proceso:

$$
\begin{aligned}
\log \left(h_{t}^{2}\right)= & C_{1}+C_{2}\left|\varepsilon_{(t-1)} / h_{(t-1)}\right|+C_{3}\left(\varepsilon_{(t-1)} / h_{(t-1)}\right) \\
& +C_{4} \log \left(h_{(t-1)}^{2}\right)
\end{aligned}
$$

Conforme puede ser observado, para mejorar la eficiencia en la estimación de la ecuación [3], se asume que los residuales $\varepsilon_{t}$ pueden ser modelados como un proceso EGARCH $(1,1)$. De acuerdo con Engle (1982), los modelos GARCH son útiles porque permiten tomar en consideración las características de series de tiempo derivadas de los mercados financieros como las tasas de inflación compensadas, las cuales por lo general tienen una distribución

1 Las tasas de interés a 30 días no deberían variar en el momento de hacerse los anuncios de la tasa de interés del banco central, toda vez que ellas incorporan toda la información disponible en los mercados y los agentes buscan anticiparse a las decisiones del banco central. Es decir, las tasas a 30 días solo varían si en los anuncios de la tasa de política existe alguna sorpresa de política o un componente inesperado. Por tanto, este componente es el que tiene potencial para afectar los mercados y las expectativas de inflación. 
que no es normal y muestran una alta curtosis. Concretamente, el modelo GARCH exponencial (EGARCH) es un modelo propuesto por Nelson (1991) y permite diferenciar los efectos asimétricos sobre las expectativas de inflación de los choques positivos y negativos. Esta es una metodología estadística utilizada de forma amplia en la literatura sobre la comunicación de los bancos centrales (Blinder et al., 2008).

Como variables control son utilizados datos de variables macroeconómicas y financieras, puesto que estas variables permiten explicar la dinámica de las expectativas de inflación (Mankiw et al., 2003). La volatilidad de los mercados financieros mundiales afecta el desempeño de las economías emergentes $\mathrm{y}$, de forma indirecta, la formación de expectativas (Kennedy \& Palerm, 2014). El índice VIX es un indicador de volatilidad del mercado de opciones de Chicago y mide el riesgo del mercado financiero de los Estados Unidos. Por tanto, como una primera variable control son utilizadas las variaciones del índice VIX $(\Delta V I X)$. De acuerdo con Égert (2009), en el caso de economías emergentes, los precios de los commodities afectan el desempeño financiero y macroeconómico. En el caso de Colombia, las exportaciones dependen del sector primario, en el cual el petróleo representa casi el $50 \%$ del total de exportaciones (López,
Montes, Garavito \& Collazos, 2013). Basados en esto, la segunda variable control son los cambios en el precio del petróleo ( $\triangle O i l)$. Hay evidencia de que en el caso de la economía colombiana existe un pass-through de la tasa de cambio sobre los precios internos, que es incompleto, asimétrico y no lineal (Rincón \& Rodríguez, 2016). En consecuencia, como una tercera variable control es utilizada los cambios en la tasa de cambio $(\Delta e)$.

En suma, son consideradas tres variables control $(\Delta V I X, \Delta O i l, \Delta e)$. Los datos macroeconómicos y financieros son normalizados por su desviación estándar en la construcción de cada serie de tiempo. Con base en información disponible en el sitio web del Banco de la República de Colombia para los títulos públicos nominales e indexados, los datos usados en este estudio tienen periodicidad mensual y comprenden el período de enero de 2008 hasta diciembre de 2016. A pesar de que existe información sobre las tasas de interés de los títulos públicos en UVR y en pesos desde el año 2003, el análisis comienza en 2008 porque desde este año existe mayor estabilidad en la meta de inflación del banco central, luego de un proceso desinflacionario que comenzó desde finales de 1990. Entre 2000 - 2007 ocurrieron siete cambios en la meta de inflación del banco central, lo que ocasionó mayor volatilidad financiera en los mercados 
en cuanto los agentes aprendían sobre los objetivos a largo plazo del banco. Sumado a esto, para generar mayor estabilidad, desde el año 2008 el banco central comenzó a publicar las minutas de la última reunión de la Junta Directiva del Banco de la República como un esfuerzo institucional por explicar a los mercados la forma en que se conduce la política monetaria; además, la meta de inflación no ha vuelto a cambiar desde el año 2010. En suma, en el período 2008-2016 es posible verificar una mayor estabilidad en los objetivos del banco central que posibilitan evaluar mejor los efectos atribuibles a los anuncios del banco sobre las expectativas.

La fuente de los datos y las estadísticas descriptivas son presentadas en el Anexo 1. Como es usual, el utilizar series de tiempo lleva a la necesidad de verificar si las series tienen raíz unitaria para evitar la posibilidad de regresiones espurias. Por tanto, el test Dickey-Fuller Aumentado (ADF), el test Phillips-Perron (PP) y el test Kwiatkowski-Phillips-Schmidt-Shin (KPSS) fueron desarrollados. Los resultados están en el Anexo 2 en el anexo y es posible verificar que todas las series por utilizar son integradas de orden cero $(\mathrm{I}(0))$.

\section{EVIDENCIA EMPÍRICA}

Los resultados de las estimaciones son presentados en la Tabla 1. Cada columna reporta el resultado de la regresión de cambios en las expectativas frente a los anuncios de política monetaria, y se agrega cada variable control al tiempo.

Tabla 1. Efectos de la comunicación del banco central sobre las expectativas de inflación (EGARCH $(1,1))$

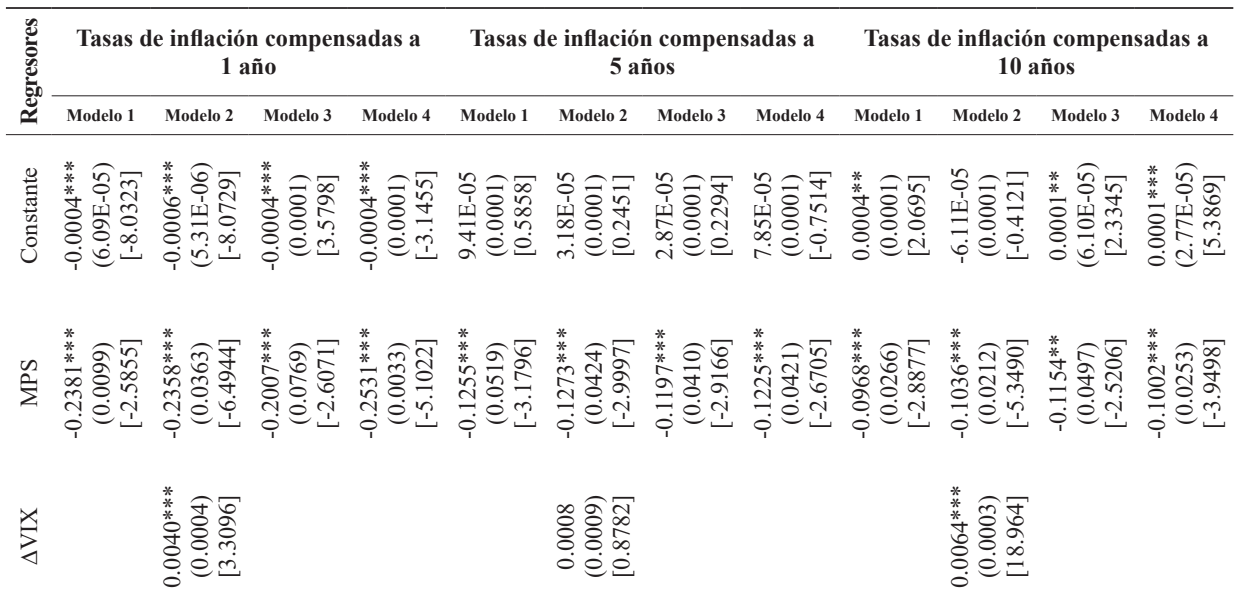


Continuación Tabla 1.

\begin{tabular}{|c|c|c|c|c|c|c|c|c|c|c|c|c|}
\hline \multirow{2}{*}{ 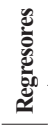 } & \multicolumn{4}{|c|}{$\begin{array}{l}\text { Tasas de inflación compensadas a } \\
1 \text { año }\end{array}$} & \multicolumn{4}{|c|}{$\begin{array}{l}\text { Tasas de inflación compensadas a } \\
5 \text { años }\end{array}$} & \multicolumn{4}{|c|}{$\begin{array}{l}\text { Tasas de inflación compensadas a } \\
10 \text { años }\end{array}$} \\
\hline & Modelo 1 & Modelo 2 & Modelo 3 & Modelo 4 & Modelo 1 & Modelo 2 & Modelo 3 & Modelo 4 & Modelo 1 & Modelo 2 & Modelo 3 & Modelo 4 \\
\hline $\bar{\sigma}$ & & & 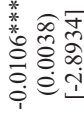 & & & & 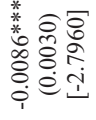 & & & & 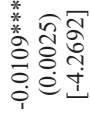 & \\
\hline 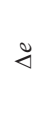 & & & & 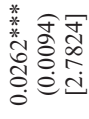 & & & & 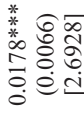 & & & & 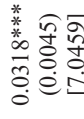 \\
\hline 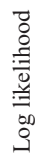 & 522.58 & 525.92 & 526.93 & 525.88 & 541.91 & 552.18 & 553.07 & 566.51 & 485.55 & 495.43 & 538.04 & 535.06 \\
\hline ô & 108 & 108 & 108 & 108 & 108 & 108 & 108 & 108 & 108 & 108 & 108 & 108 \\
\hline
\end{tabular}

Nota: niveles marginales de significancia: $(* * *)$ denota $0.01,(* *)$ denota $0.05, \mathrm{y}(*)$ denota 0.10 . Errores estándar entre paréntesis y estadísticas t en corchetes. Obs= número de observaciones.

Los resultados de las estimaciones en la Tabla 1 permiten observar que las expectativas de inflación responden al componente inesperado de los anuncios de la tasa de política monetaria. En todos los modelos, el coeficiente asociado con los anuncios es negativo y significativo. Para el período 20082016, los resultados sugieren que una variación del coeficiente asociado al componente inesperado de los anuncios de la tasa de interés (MPS) disminuye entre 0.20 y 0.25 puntos básicos las tasas de inflación compensadas a un año. Para las tasas a 5 años, la respuesta es de 0.12 puntos básicos y para las tasas de inflación a 10 años la respuesta es alrededor de 0.10 puntos básicos. Es decir, los efectos de los anuncios son decrecientes para las expectativas de inflación a largo plazo. Un resultado usual en la literatura empírica (Moessner, 2015). Es importante señalar que el banco central cambia su tasa de política cuando las expectativas están desviadas de la meta para señalizar al mercado su compromiso con el control de la inflación. En consecuencia, acorde con los resultados, hay evidencia de que los anuncios del banco central tienen la fuerza para guiar las expectativas hacia la meta. Un resultado similar es reportado por Kliesen y Schmid (2004), Gürkaynak et al. (2010) y Moessner (2015).

Si las expectativas están bien ancladas, ellas no deberían responder a los datos 
macroeconómicos y financieros (Gürkaynak et al., 2010). No obstante, en el caso de la economía colombiana se encuentra que los parámetros asociados con las variables control son todos significativos en términos estadísticos y contienen información relevante para el comportamiento de las expectativas de inflación. Por tanto, los choques macroeconómicos y financieros son un riesgo para la estabilidad de precio $y$ afectan la confianza en la meta de inflación en Colombia.

En el caso del riesgo internacional, el parámetro es positivo y significativo. Así, la volatilidad financiera en los mercados mundiales desestabiliza las expectativas de inflación en Colombia. Un resultado similar es reportado por Galati et al. (2011) para el período posterior a la crisis financiera de 2008 en el caso de las expectativas de inflación en Estados Unidos. Sumado a esto, De Pooter et al. (2014) encuentran un resultado similar para el caso de Brasil y México. El parámetro asociado con la tasa de cambio es positivo y significativo, lo cual indica que la devaluación incrementa las expectativas de inflación en Colombia. Este resultado confirma el pass-through de la tasa de cambio sobre los precios domésticos (Rincón \& Rodríguez, 2016). Por último, respecto a los precios del petróleo se observa que las variaciones de este precio provocan un efecto negativo y significativo sobre las expectativas de inflación. En el caso de la economía colombiana, una parte importante de la canasta básica depende de los bienes importados y su costo está conectado con los precios del petróleo. Por tanto, un mayor precio del petróleo al señalizar mayores entradas de divisas ayuda a anclar las expectativas de inflación por causa de la disminución futura en el precio de la canasta básica. Con relación a este último resultado, De Pooter et al. (2014) encuentran una evidencia empírica similar para el caso de México.

\section{EXPECTATIVAS DE INFLACIÓN Y CREDIBILIDAD DE LA POLÍTICA MONETARIA}

Cuando la política del banco central es creíble, las expectativas tendrían que estar bien ancladas en la meta y no deberían desestabilizarse frente a los choques macroeconómicos y financieros (Jansen \& De Haan, 2007). A pesar de esto, hay pocos estudios que suministren evidencia del vínculo entre los anuncios del banco central, el escenario de credibilidad y las expectativas de inflación.

La novedad de este estudio es el análisis del escenario de credibilidad en el cual son hechos los anuncios del banco central. Entre los índices que existen para medir la credibilidad, el índice propuesto por De Mendonça (2007) se ajusta bien al caso de economías emergentes que han adoptado el régimen de 
metas de inflación. El índice que se va a construir lleva en consideración la desviación de las expectativas de inflación o inflación esperada con relación a la meta central de inflación. Se propone que la política monetaria tiene credibilidad total $(C R E D=1)$ si las expectativas de inflación del público $(E(\pi))$ coinciden con la meta central de inflación del banco central $\left(\pi \_t\right)$. La credibilidad es cero $(C R E D=0)$ si las expectativas están arriba o abajo de los límites de tolerancia para la inflación. Dado que los dos casos anteriores son casos extremos, el índice toma un valor entre 0 y 1 cuando las expectativas se sitúan en los intervalos de tolerancia para la inflación. O sea, el índice se encuentra normalizado entre 0 y 1 .
Donde $E(\pi)$ son las expectativas de inflación, $\pi_{\mathrm{t}}$ es la meta central de inflación del banco, $\pi_{t M I N^{*}} \mathrm{y} \pi_{t M A X^{*}}$ representan los límites mínimo y máximo de la meta de inflación para cada año.

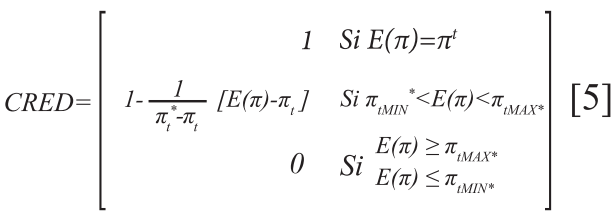

Con base en datos mensuales del período 2008-2016, el comportamiento de las expectativas de inflación, calculadas por medio de las tasas de inflación compensadas a un año, y las metas de inflación, variables esenciales para construir el índice de credibilidad, son presentadas en la Figura 2.

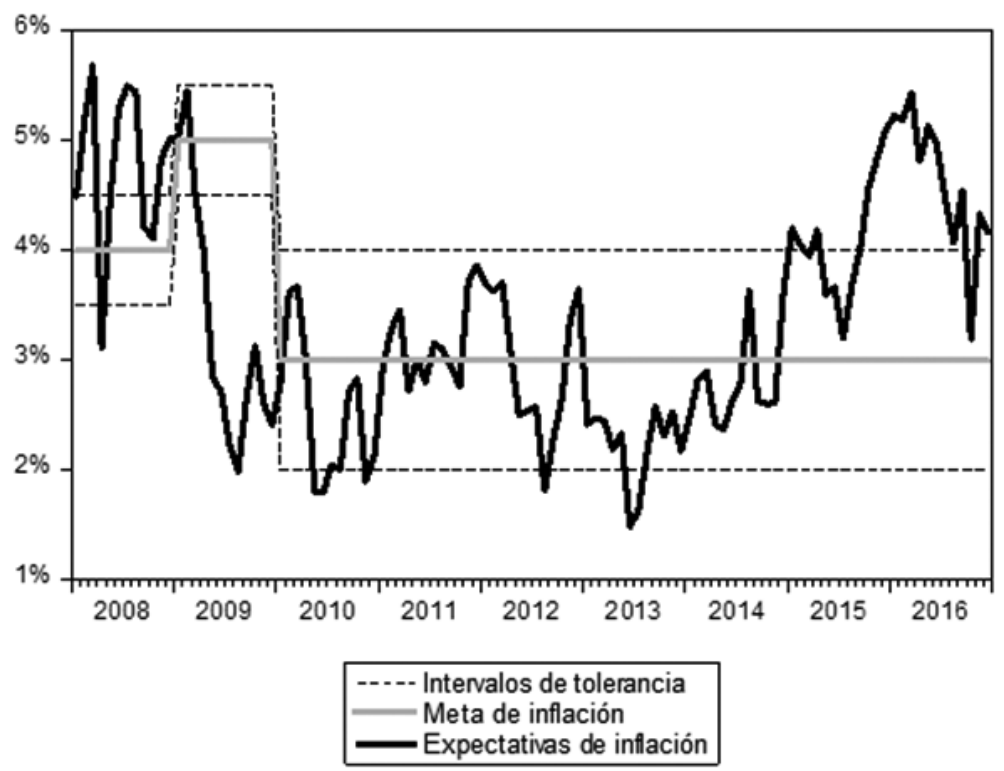

Figura 2. Expectativas de inflación, intervalos de tolerancia y meta de inflación. Fuente: elaboración propia con base en datos del banco central de Colombia. 
Con base en los datos presentados en la Figura 2 es construido el índice de credibilidad. La Figura 3 muestra el desempeño de la credibilidad durante el período 2008-2016. Como era de esperarse, existen diferencias en la trayectoria. El comportamiento del índice permite identificar dos etapas o fases en el período. Una primera etapa - "periodo de desarrollo" (enero de 2008 a noviembre de 2012) - es marcada por un período de desinflación que traía la economía colombiana desde comienzos del siglo XXI. En este período, la credibilidad fue cercana a cero en 2008 y luego comenzó a crecer cuando el banco central logró anclar de formar momentánea las expectativas de inflación en los intervalos de tolerancia. En este período, la media de la credibilidad fue de 0.29 . La segunda etapa - "período de inestabilidad" (diciembre de 2012 a diciembre de 2016) - es caracterizada por un incremento en las expectativas de inflación a causa de algunos choques externos, como la caída del precio del petróleo y la devaluación. En este período, la media de la credibilidad fue de 0.43 .

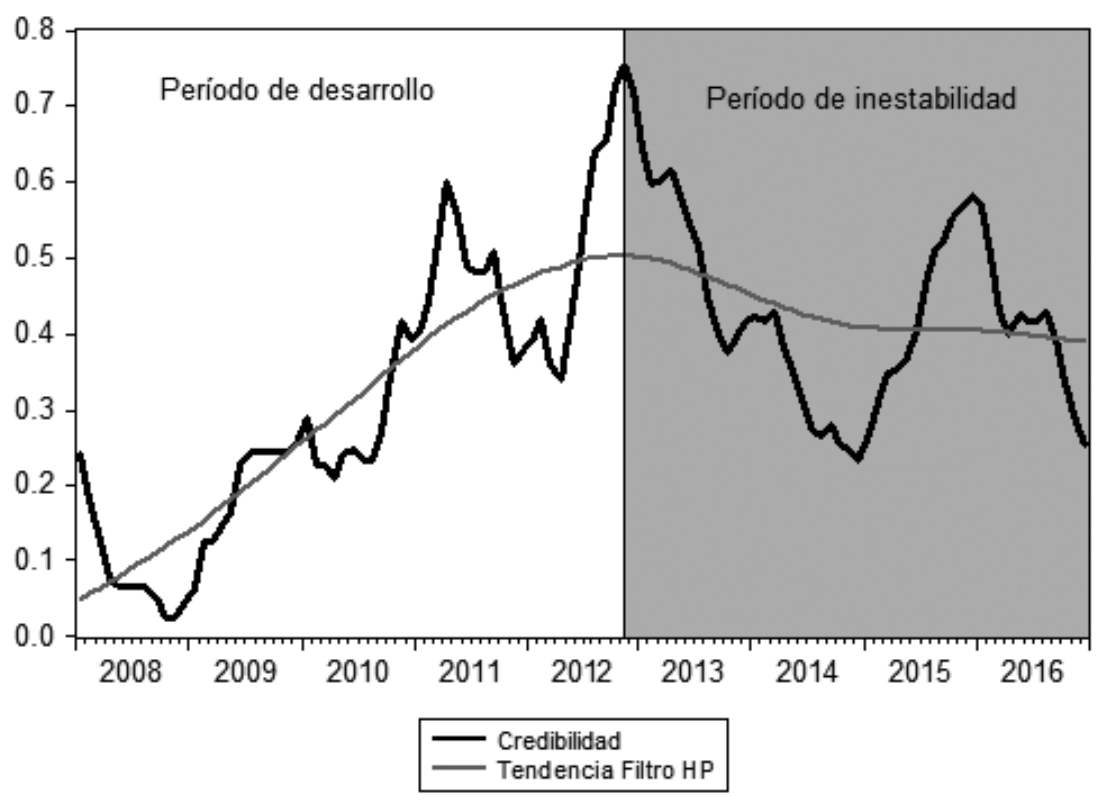

F-statistic

Chow Breakpoint Test 2012M11

Log likelihood ratio

7.54

Prob. $\mathrm{F}(1,105)$

0.006

Prob. Chi-Square(1)

0.006

Figura 3. Credibilidad de la política monetaria en Colombia.

Fuente: elaboración propia con base en datos del banco central de Colombia. 
Una vez que existe un quiebre estructural de la credibilidad de la política monetaria en el tiempo, es posible verificar si hay alguna diferencia en el impacto de los anuncios de la tasa de políticas y de los choques macroeconómicos y financieros sobre el anclaje de las expectativas. Por tanto, con la misma metodología adoptada en la sección previa, son reestimadas las ecuaciones (2) y (3) solo con las expectativas de inflación a un año, ya que la credibilidad fue medida con expectativas anuales. Son considerados dos escenarios básicos: el período de desarrollo y el período de inestabilidad. Las estimaciones son presentadas en la Tabla 2.

Tabla 2. Efectos de la comunicación del banco central sobre las expectativas de inflación a un año por fases de la credibilidad

\begin{tabular}{|c|c|c|c|c|c|c|c|c|}
\hline \multirow{2}{*}{$\begin{array}{c}\text { Regre- } \\
\text { sores }\end{array}$} & \multicolumn{4}{|c|}{ Período de desarrollo 2008-2012m11 } & \multicolumn{4}{|c|}{ Período de inestabilidad 2012m12-2016 } \\
\hline & Modelo 1 & Modelo 2 & Modelo 3 & Modelo 4 & Modelo 1 & Modelo 2 & Modelo 3 & Modelo 4 \\
\hline 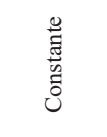 & 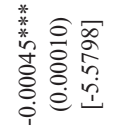 & 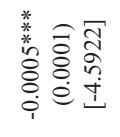 & 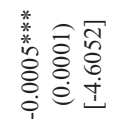 & 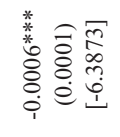 & 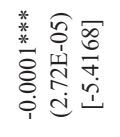 & 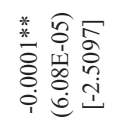 & 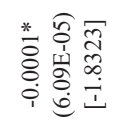 & 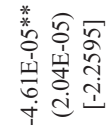 \\
\hline$\hat{\Sigma}_{\Sigma}^{\infty}$ & 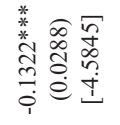 & 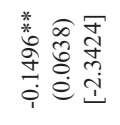 & 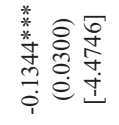 & 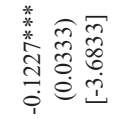 & 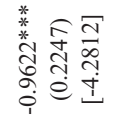 & 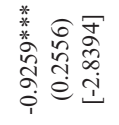 & 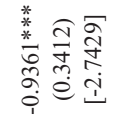 & 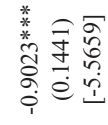 \\
\hline 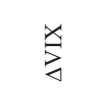 & & 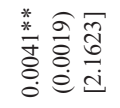 & & & & 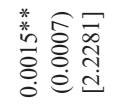 & & \\
\hline $\bar{\sigma}$ & & & 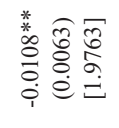 & & & & 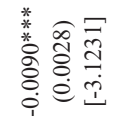 & \\
\hline$\ddot{4}$ & & & & 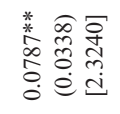 & & & & 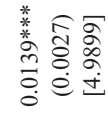 \\
\hline $\begin{array}{l}\text { Log } \\
\text { likelihood }\end{array}$ & 269.79 & 271.14 & 272.58 & 270.12 & 260.61 & 263.66 & 265.84 & 266.95 \\
\hline Obs. & 59 & 59 & 59 & 59 & 49 & 49 & 49 & 49 \\
\hline
\end{tabular}

Nota: niveles marginales de significancia: $(* * *)$ denota $0.01,(* *)$ denota 0.05 , y $(*)$ denota 0.10 . Errores estándar entre paréntesis y estadísticas t en corchetes. Obs $=$ número de observaciones.

Los resultados de la Tabla 2 indican que los anuncios del banco central ayudaron a administrar las expectativas de inflación en la dirección correcta en ambos períodos. Sin embargo, la capacidad del banco central para afectar el anclaje de las expectativas fue diferente, dado el comportamiento de la 
credibilidad. Los resultados muestran que la sensibilidad de las expectativas de inflación a los anuncios aumentó en el segundo período. En concreto, la reacción de las expectativas de inflación a los anuncios (el coeficiente asociado a la variable MPS) fue mayor en el período de diciembre de 2012 a diciembre de 2016 , con relación al período de enero de 2008 a noviembre de 2012, con un efecto casi un $80 \%$ más alto. Como resultado, en el período 2012-2016 los anuncios del banco central fueron más efectivos en anclar las expectativas de inflación en la meta anunciada.

Los coeficientes asociados con los choques macroeconómicos y financieros muestran significancia estadística en ambos períodos. Por tanto, la hipótesis de que la credibilidad de la política monetaria no es total es confirmada. A pesar de esto, los resultados sugieren que cuando la credibilidad estuvo mejor construida (período 2012-2016), los choques macroeconómicos desestabilizaron las expectativas de inflación en menor extensión. En consonancia con esto, los coeficientes de las variables control fueron menores en el período 2012-2016 con relación al período 2008-2012. En suma, la evidencia indica que la credibilidad es fundamental para analizar el anclaje de las expectativas. Esta perspectiva teórica, empíricamente no verificada para economías emergentes, es apuntada por Jansen y De Haan (2007), Gürkaynak et al. (2010), y Galati et al. (2011).

\section{CONCLUSIONES}

Este documento estudió el impacto de los anuncios de política monetaria sobre las expectativas de inflación para Colombia, una economía emergente que opera bajo el esquema de inflación objetivo. Los resultados de este estudio permiten hacer tres observaciones. Primera, los anuncios del banco central tienen los efectos apropiados sobre el anclaje de las expectativas de inflación en Colombia. Segundo, las expectativas de inflación en Colombia no están ancladas con firmeza. Tercero, es importante considerar el escenario de credibilidad cuando se analizan los factores que afectan las expectativas. En concreto, la habilidad del banco central para afectar las expectativas es mayor cuando la credibilidad de la política es más alta. Sumado a esto, conforme señalan en términos teóricos Gürkaynak et al. (2010), una credibilidad más alta de la política monetaria permite que las expectativas de inflación sean más estables ante los choques externos.

\section{AGRADECIMIENTOS}

Los autores agradecen los comentarios y sugerencias de los evaluadores anónimos. Así mismo, asumen la responsabilidad por las opiniones contenidas en el presente artículo. 


\section{REFERENCIAS}

Bernanke, B., Laubach, T., Mishkin, F. \& Posen, A. (1999). Inflation Targeting: Lessons from the International Experience. Princeton, NJ: Princeton University Press.

Blinder, A., Ehrmann, M., Fratzcher, M., De Haan, J. \& Jansen, D. (2008). Central Bank Communication and Monetary Policy. Journal of Economic Literature, 46(4), 910-945.

Born, B., Ehrmann, M. \& Fratzscher, M. (2014). Central Bank Communication on Financial Stability. The Economic Journal, 124(577), 701-734.

Ciro, J.C. \& De Mendonça, H.F. (2017). Effect of Credibility and Reputation on Discretionary Fiscal Policy: Empirical Evidence from Colombia. Empirical Economics, 53(4), 1529-1552.

Clark, T. \& Nakata, T. (2008). Has the Behavior of Inflation and Long-Term Inflation Expectations Changed? Economic Review Federal Reserve Bank of Kansas City, 93(1), 17-49.

De Mendonça, H. F. (2007). Towards Credibility from Inflation Targeting: The Brazilian Experience. Applied Economics, 39(20), 2599-2615.

De Pooter, M., Robitaille, P., Walker, I. \& Zdinak, M. (2014). Are Long-Term Inflation Expectations Well Anchored in Brazil, Chile and Mexico? International Journal of Central Banking, 10(2), 337-400.

Ehrmann, M. \& Fratzscher, M. (2007). Communication and Decision-Making by Central Bank Committees. Different Strategies, Same Effectiveness. Journal of Money, Credit and Banking, 39(3), 509-541.

Ehrmann, M., Fratzscher, M. Gürkaynak, R. \& Swanson, E. (2011). Convergence and Anchoring of Yield Curves in the Euro Area. The Review of Economics and Statistics, 93(1), 350-364.

Égert, B. (2009). The Impact of Monetary and Commodity Fundamentals, Macro News and Central Bank Communication on the Exchange Rate: Evidence from South Africa. Open Economies Review, 21(5), 655-677.

Eijffinger, S. \& Geraats, P. (2006). How Transparent Are Central Banks? European Journal of Political Economy, 22(1), 1-21. 
Engle, R. (1982). Autoregressive Conditional Heteroscedasticity with Estimates of the Variance of United Kingdom Inflation. Econometrica, 50(4), 987-1008.

Galati, G. Poelhekke, S. \& Zhou, C. (2011). Did the Crisis Affect Inflation Expectations? International Journal of Central Banking, 7(1), 167-207.

Gürkaynak, R., Levin, A. \& Swanson, E. (2010). Does Inflation Targeting Anchor Long-Run Inflation Expectations? Evidence from Long-Term Bond Yields in the U.S., U.K., and Sweden. Journal of the European Economic Association, 8(6), 1208-1242.

Jansen, D. J. (2011). Does the Clarity of Central Bank Communication Affect Volatility in Financial Markets? Evidence from Humphrey-Hawkins Testimonies. Contemporary Economic Policy, 29(4), 494-509.

Jansen, D. \& De Haan, D. (2007). The Importance of Being Vigilant: Has ECB Communication Influenced Euro Area Inflation Expectations? CESifo Working Paper Series No. 2134 (pp.1-29). Munich.

Johnson, D. (2003). The Effect of Inflation Targets on the Level of Expected Inflation in Five Countries. Review of Economics and Statistics, 85(4), 1076-81.

Kennedy, M. \& Palerm, A. (2014). Emerging Market Bond Spreads: The Role of Global and Domestic Factors from 2002 to 2011. Journal of International Money and Finance, 43(1), 70-87.

Kliesen, K. \& Schmid, F. (2004). Monetary Policy Actions, Macroeconomic Data Releases, and Inflation Expectations. Federal Reserve Bank of St Louis Review, 86(3), 9-21.

Kuttner, K. (2001). Monetary Policy Surprises and Interest Rates: Evidence from the FED FUNDS Futures Market. Journal of Monetary Economics, 47(3), 523-544.

Levieuge, G., Lucotte, Y. \& Ringuedé. S. (2015). Central Bank Credibility and the Expectations Channel: Evidence Based on a New Credibility Index. National Bank of Poland Working Papers 209 (pp. 1-45). Warsaw.

Levin, A., Natalucci, F. \& Piger, J. (2004). The Macroeconomic Effects of Inflation Targeting. Federal Reserve Bank of St. Louis Review, 86(4), 51-80. 
López E., Montes, E., Garavito, A. \& Collazos, M. (2013). La economía petrolera en Colombia. Relaciones intersectoriales e importancia en la economía nacional. Borradores de Economía, (566), 1-56.

Mankiw, N., Reis, R. \& Wolfers, J. (2003). Disagreement about Inflation Expectations. NBER Macroeconomics Annual, 18, 209-268.

Moessner, R. (2015). Reactions of Real Yields and Inflation Expectations to Forward Guidance in the United States. Applied Economics, 47(26), 2671-2682.

Montes, G. (2013). Credibility and Monetary Transmission Channels under Inflation Targeting: An Econometric Analysis from a Developing Country. Economic Modelling, 30, 670-684.

Montes, G., Oliveira, L., Curi, A. \& Nicolay, R. (2016). Effects of Transparency, Monetary Policy Signalling and Clarity of Central Bank Communication on Disagreement about Inflation Expectations. Applied Economics, 48(7), $1-18$.

Nelson, D. (1991). Conditional Heteroskedasticity in Asset Returns: A New Approach. Econometrica, 59(2), 349-380.

Rincón, H. \& Rodríguez, N. (2016). Pass-Through of Exchange Rate Shocks on Inflation: A Bayesian Smooth Transition VAR Approach. Borradores de Economía, (930), 1-75.

Vargas, H., González, A., González, E., Romero, J. \& Rojas, L. (2010, August). Assessing Inflationary Pressures in Colombia. BIS Papers, Bank for International Settlements, No. 49 (pp.129-171). Basel.

Woodford, M. (2005). Central Bank Communication and Policy Effectiveness. NBER Working Paper N. ${ }^{\circ} 11898$, (pp. 1-67). Cambridge. 


\section{ANEXOS}

Anexo 1. Datos y fuente

\begin{tabular}{cll}
$\begin{array}{c}\text { Nombre de la } \\
\text { variable }\end{array}$ & \multicolumn{1}{c}{ Descripción } & \multicolumn{1}{c}{ Fuente } \\
\hline $\boldsymbol{f}_{\text {nominal }}$ & Retorno de los bonos nominales & $\begin{array}{l}\text { Banco de la República de Colombia } \\
\text { http://www.banrep.gov.co/es/tes }\end{array}$ \\
\hline $\boldsymbol{f}_{\text {index }}$ & Retorno de los bonos indexados & $\begin{array}{l}\text { Banco de la República de Colombia } \\
\text { http://www.banrep.gov.co/es/tes }\end{array}$ \\
\hline $\boldsymbol{C R E D}$ & $\begin{array}{l}\text { Índice de credibilidad de la política } \\
\text { monetaria. }\end{array}$ & Desarrollada por los autores \\
\hline $\boldsymbol{\pi} \boldsymbol{t}$ & Meta de inflación del banco central & $\begin{array}{l}\text { Banco de la República de Colombia } \\
\text { http://w w w.b a n r e p.g o v.co/e s/ } \\
\text { meta-inflacion }\end{array}$ \\
\hline $\boldsymbol{M P S}$ & Calculada con la tasa IBR a 30 días & $\begin{array}{l}\text { Banco de la República de Colombia } \\
\text { (http://www.banrep.gov.co/es/ibr) }\end{array}$ \\
\hline $\boldsymbol{O}$ & $\begin{array}{l}\text { Tipo de cambio del peso colombiano/ } \\
\text { dólar americano }\end{array}$ & $\begin{array}{l}\text { Banco de la República de Colombia } \\
\text { (http://www.banrep.gov.co/es/trm) }\end{array}$ \\
\hline $\boldsymbol{V I X}$ & Precio del petróleo referencia WTI & $\begin{array}{l}\text { Reserva Federal de St. Louis (http://re- } \\
\text { search.stlouisfed.org/) }\end{array}$ \\
\hline & $\begin{array}{l}\text { Volatilidad del índice de mercado de } \\
\text { valores S\&P 500. }\end{array}$ & $\begin{array}{l}\text { Reserva Federal de St. Louis (http://re- } \\
\text { search.stlouisfed.org/) }\end{array}$ \\
\hline
\end{tabular}

Fuente: elaboración propia.

Anexo 2. Estadísticas descriptivas

\begin{tabular}{cccccc}
\hline Variable & Media & Min. & Max. & SD & Curtosis \\
\hline$\Delta \boldsymbol{\pi}^{e}{ }_{(1-\text { year })}$ & -0.0003 & -0.0226 & 0.0117 & 0.0031 & 25.6173 \\
$\Delta \boldsymbol{\pi}^{e}{ }_{(5-\text { year) }}$ & -0.0005 & -0.0094 & 0.0058 & 0.0020 & 8.1889 \\
$\Delta \boldsymbol{\pi}^{e}{ }_{(10-\text {-year })}$ & -0.0004 & -0.0299 & 0.0152 & 0.0047 & 21.8245 \\
$\boldsymbol{M P S}$ & $-1.01 \mathrm{E}-05$ & -0.0024 & 0.0024 & 0.0003 & 38.4911 \\
$\Delta \boldsymbol{e}$ & 0.0006 & -0.0250 & 0.0340 & 0.0088 & 4.8760 \\
$\Delta \boldsymbol{O}$ oil & 0.0013 & -0.0924 & 0.1783 & 0.0311 & 12.3307 \\
$\Delta \boldsymbol{V I X}$ & 0.0199 & -0.1851 & 0.4534 & 0.0862 & 9.7588 \\
\hline
\end{tabular}

Fuente: elaboración propia. 
Anexo 3. Los test de raíz unitaria (ADF, PP, y KPSS)

\begin{tabular}{|c|c|c|c|c|c|c|c|c|c|c|c|c|}
\hline \multirow[b]{2}{*}{ Series } & \multicolumn{4}{|c|}{ ADF } & \multicolumn{4}{|c|}{ PP } & \multicolumn{4}{|c|}{ KPSS } \\
\hline & Lags & Esp. & Test & $\begin{array}{l}\text { C. V } \\
(5 \%)\end{array}$ & Band & Esp. & Test & $\begin{array}{l}\text { C. V } \\
(5 \%)\end{array}$ & Band & Esp. & Test & $\begin{array}{r}\text { C. V } \\
(5 \%)\end{array}$ \\
\hline$\Delta \pi^{e}{ }_{1}$ & 0 & $\mathrm{~N}$ & -9.31 & -1.94 & 4 & $\mathrm{~N}$ & -9.33 & -1.94 & 2 & $\mathrm{C}, \mathrm{T}$ & 0.08 & 0.14 \\
\hline$\Delta \boldsymbol{\pi}^{e}{ }_{5}$ & 1 & $\mathrm{C}, \mathrm{T}$ & -6.98 & -3.45 & 3 & $\mathrm{C}, \mathrm{T}$ & -11.99 & -3.45 & 2 & $\mathrm{C}, \mathrm{T}$ & 0.05 & 0.14 \\
\hline$\Delta \pi^{e}{ }_{10}$ & 1 & $\mathrm{~N}$ & -5.84 & -1.94 & 5 & $\mathrm{~N}$ & -12.21 & -1.94 & 3 & $\mathrm{C}$ & 0.20 & 0.46 \\
\hline$M P S$ & 0 & $\mathrm{~N}$ & -10.08 & -1.94 & 4 & $\mathrm{~N}$ & -10.08 & -1.94 & 4 & $\mathrm{C}$ & 0.10 & 0.46 \\
\hline$\Delta e$ & 0 & $\mathrm{~N}$ & -13.28 & -1.94 & 9 & $\mathrm{~N}$ & -16.57 & -1.94 & 6 & $\mathrm{C}$ & 0.15 & 0.46 \\
\hline$\Delta O$ il & 0 & $\mathrm{~N}$ & -11.32 & -1.94 & 2 & $\mathrm{~N}$ & -11.28 & -1.94 & 3 & $\mathrm{C}$ & 0.36 & 0.46 \\
\hline$\Delta V I X$ & 0 & $\mathrm{~N}$ & -8.93 & -1.94 & 3 & $\mathrm{~N}$ & -9.01 & -1.94 & 1 & $\mathrm{C}$ & 0.40 & 0.46 \\
\hline \multicolumn{13}{|c|}{ Período de desarrollo } \\
\hline$\Delta \pi^{e}{ }_{1}$ & 0 & $\mathrm{~N}$ & -8.07 & -1.94 & 0 & $\mathrm{~N}$ & -6.57 & -1.94 & 1 & $\mathrm{C}$ & 0.26 & 0.46 \\
\hline MPS & 0 & $\mathrm{~N}$ & -2.83 & -1.94 & 2 & $\mathrm{~N}$ & -2.79 & -1.94 & 5 & $\mathrm{C}, \mathrm{T}$ & 0.09 & 0.14 \\
\hline$\Delta e$ & 0 & $\mathrm{~N}$ & -8.95 & -1.94 & 3 & $\mathrm{~N}$ & -9.09 & -1.94 & 4 & $\mathrm{C}$ & 0.13 & 0.46 \\
\hline$\Delta O i l$ & 2 & $\mathrm{~N}$ & -4.50 & -1.94 & 1 & $\mathrm{~N}$ & -8.47 & -1.94 & 5 & $\mathrm{C}$ & 0.04 & 0.46 \\
\hline$\Delta V I X$ & 0 & $\mathrm{~N}$ & -6.89 & -1.94 & 3 & $\mathrm{~N}$ & -6.87 & -1.94 & 2 & $\mathrm{C}$ & 0.20 & 0.46 \\
\hline \multicolumn{13}{|c|}{ Período de inestabilidad } \\
\hline$\Delta \pi_{1}^{e}$ & 0 & $\mathrm{~N}$ & -8.24 & -1.94 & 1 & $\mathrm{~N}$ & -8.26 & -1.94 & 4 & $\mathrm{C}$ & 0.19 & 0.46 \\
\hline MPS & 0 & $\mathrm{~N}$ & -21.85 & -1.94 & 3 & $\mathrm{~N}$ & -19.00 & -1.94 & 1 & $\mathrm{C}, \mathrm{T}$ & 0.11 & 0.14 \\
\hline$\Delta e$ & 0 & $\mathrm{~N}$ & -10.76 & -1.94 & 9 & $\mathrm{~N}$ & -11.31 & -1.94 & 9 & $\mathrm{C}$ & 0.20 & 0.46 \\
\hline$\Delta O$ il & 0 & $\mathrm{~N}$ & -7.30 & -1.94 & 2 & $\mathrm{~N}$ & -7.32 & -1.94 & 2 & $\mathrm{C}$ & 0.41 & 0.46 \\
\hline$\Delta V I X$ & 0 & $\mathrm{C}$ & -6.52 & -2.92 & 1 & $\mathrm{C}$ & -6.52 & -2.92 & 0 & $\mathrm{C}$ & 0.14 & 0.46 \\
\hline
\end{tabular}

Nota: $C . V .=$ valor crítico. Tendencia $(T), y / o$ constante $(C)$, o Ni tendencia ni Constante $(N)$ son incluidas con base en el criterio de información de Schwarz. El test KPSS fue calculado con banda Newey-West.

Fuente: elaboración propia. 